\title{
Environment or Outflows? New Insight into the Origin of NALs
}

\author{
Vivienne Wild ${ }^{1}$ and Paul Hewett ${ }^{2}$ \\ ${ }^{1}$ Institut d'Astrophysique de Paris, 75014 Paris, France \\ Email: wild@iap.fr \\ ${ }^{2}$ Institute of Astronomy, University of Cambridge, Cambridge CB3 0HA, UK
}

Keywords. quasars: absorption lines, galaxies: ISM

Recent detailed studies of narrow absorption line (NAL) systems in QSO spectra have revealed that at least $50 \%$ of QSOs have NALs associated with the central engine, and in most cases they are found to be outflowing. Will studies of NALs provide the much sought-after evidence for ubiquitous QSO feedback that can halt the formation of stars in galaxies? I present new results on the distribution of line-of-sight velocity offsets between $\mathrm{Mg}$ II absorbers and their background QSOs, based on a large catalogue of absorbers from SDSS DR6 and greatly improved QSO redshift estimates. The analysis reveals high velocity Mg II NALs out to at least $6000 \mathrm{~km} \mathrm{~s}^{-1}$ from the QSO, which cannot be ascribed to the clustering of local galaxies, similar to that observed for C IV absorbers. The existence of such low-ionization, high-velocity gas clouds in the intense ionixing field of the QSO suggests that we may indeed be witnessing the mechanical expulsion of gas, alongside the heating previously observed. We also find an excess of low-velocity Mg II NALs in radio-loud QSOs compared to radio-quiet QSOs, consistent with their different clustering amplitudes.
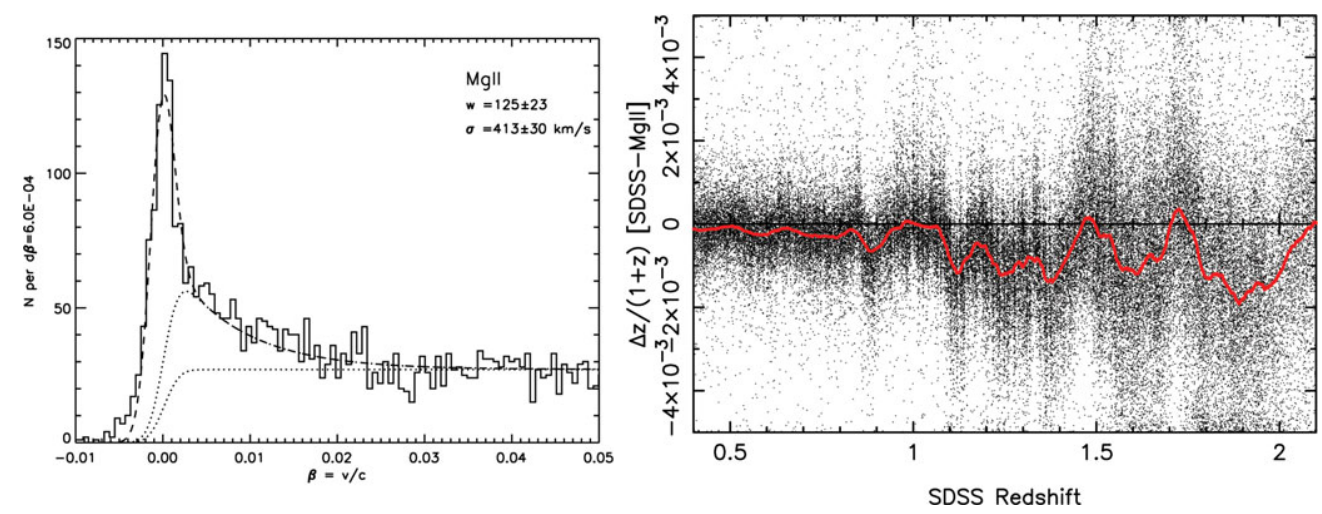

Figure 1. Left: For the first time for Mg II, we can clearly identify three populations in the distribution of velocity offsets between the $\mathrm{Mg}$ II absorption line systems and their background QSOs: (1) At large velocities, $\beta>0.02$, a constant number density due to intervening galaxies not physically associated with the QSO. (2) A clear spike at $\beta=0$ : a Gaussian distribution with mean of zero and width of a few hundred $\mathrm{km} / \mathrm{s}$. Whether these NALs primarily originate in galaxies clustered around the QSO or in the QSO host galaxy is a question we must address in the future. (3) An excess of high-velocity absorbers out to $6000 \mathrm{~km} \mathrm{~s}^{-1}$, a feature previously seen only in C IV. Right: For the analysis of $z_{\text {abs }} \sim z_{\mathrm{QSO}}$ absorption systems, accurate redshifts for the QSOs are crucial. The measurement of QSO redshifts is a difficult problem due to the substantial broadening, and sometimes centroid shifting, of the emission lines. The results presented here rely upon new SDSS QSO redshifts using a combination of available narrow emission lines and new cross-correlation templates. This figure shows strong systematics as a function of redshift in the automatic pipeline reduction of SDSS QSOs compared to the centroid of the Mg II emission line. 